\title{
Last PhD Supervised by Professor Kouyoumjian: Extended UTD by Dr. Buyukdura
}

\author{
$\underline{\text { Ayhan Altintas }}$ \\ Bilkent University, Department of Electrical and Electronics Engineering, Bilkent 06800 Ankara, Turkey \\ altintas@ee.bilkent.edu.tr
}

\begin{abstract}
While he is a Professor Emeritus at Ohio State, Professor Kouyoumjian supervised a thesis work by Merih Buyukdura. They first derived a dyadic Green's function for a PEC wedge using spherical wave functions and employed asymptotic approximation. They also derived the extended UTD in which higher order terms in the diffraction matrix are predicted. The thesis was defended in 1984. In this presentation, a brief discussion of edge waves as derived from the asymptotic expansion of dyadic Green's function in terms of spherical functions will be made and afterwards the derivation of extended UTD diffraction coefficients will be given.
\end{abstract}

\section{Introduction}

The thesis[1] by Dr. Buyukdura has mainly two parts. The first part is the derivation of dyadic Green's function for a wedge using spherical vector wave functions. An asymptotic analysis of the Green's function expansions yields the edge waves excited by the edge. This way, it rigorously derives the edge waves which were only predicted heuristically by F. Sitka [2]. In addition, using the Dyadic Green's functions, scattering from a spherical boss located on the edge has been solved. By using T-matrix method, spherical boss can be replaced by and arbitrary scatterer. Finally, the solution is extended to the case of multiple scatterers. The second part of the thesis is about the derivation of extended UTD diffraction coefficients as a modification to the well-known soft and hard diffraction coefficients. The extended UTD solution contains higher order terms by which large parameter can be reduced without sacrificing accuracy. Prof. Dr. Buyukdura passed away in 2007, so this presentation will be a commemoration of Prof. Buyukdura also.

\section{Derivation of Dyadic Green's Function for a Wedge in Spherical Coordinates}

J. B. Keller [3] and R.G. Kouyoumjian-P.H. Pathak [4] have solved the scattering form a wedge using 2D Green's function obtained in terms of cylindrical wave functions. Then by making asymptotic approximations, they derived the GTD and UTD diffraction coefficients respectively. These diffraction coefficients are then extended to 3D problems by including the angle parameter $\Theta$ in Figure 1.

The application in 3D is the result of field continuity considerations, but not derived rigorously. As the angle $\Theta$ gets smaller, the accuracy of the diffracted fields gets weaker. For this reason, Green's function for a wedge is derived in terms of spherical wave functions which rigorously satisfy the edge condition and the source singularity. The final expression for the Green's function is given as Equation (51) in [5].

To investigate the field behavior in the paraxial region, fields of a small dipole near the wedge is approximated by choosing the most significant terms in the expression due to fact that $\Theta$ being small. Furthermore, eliminating the higher order terms which are small near the edge, the following expression is found for the electric field:

$$
\bar{E}(\bar{R}) \cong K \frac{e^{-j k_{0} z}}{z^{\frac{\pi}{\gamma}} \rho^{1-\frac{\pi}{\gamma}}}\left[\hat{\rho} \sin \left(\frac{\pi}{\gamma} \phi\right)+\hat{\phi} \cos \left(\frac{\pi}{\gamma} \phi\right)\right]
$$

where $\gamma$ is the exterior wedge angle, $k_{o}$ is the free space wave number, $\rho$ is the radial distance from the $z$-axis, and $K$ is a constant. The above expression reveals that for interior wedge angles smaller than 180 degrees, there is relatively strong coupling between antennas close to the edge. Specifically, for a half-plane, this edge wave field varies as

$$
\bar{E}(\bar{R}) \cong K \frac{e^{-j k_{0} z}}{\sqrt{z} \sqrt{\rho}}\left[\hat{\rho} \sin \left(\frac{\phi}{2}\right)+\hat{\phi} \cos \left(\frac{\phi}{2}\right)\right]
$$

This behavior has been deducted before by Sitka [2] and Van Bladel [6]. 


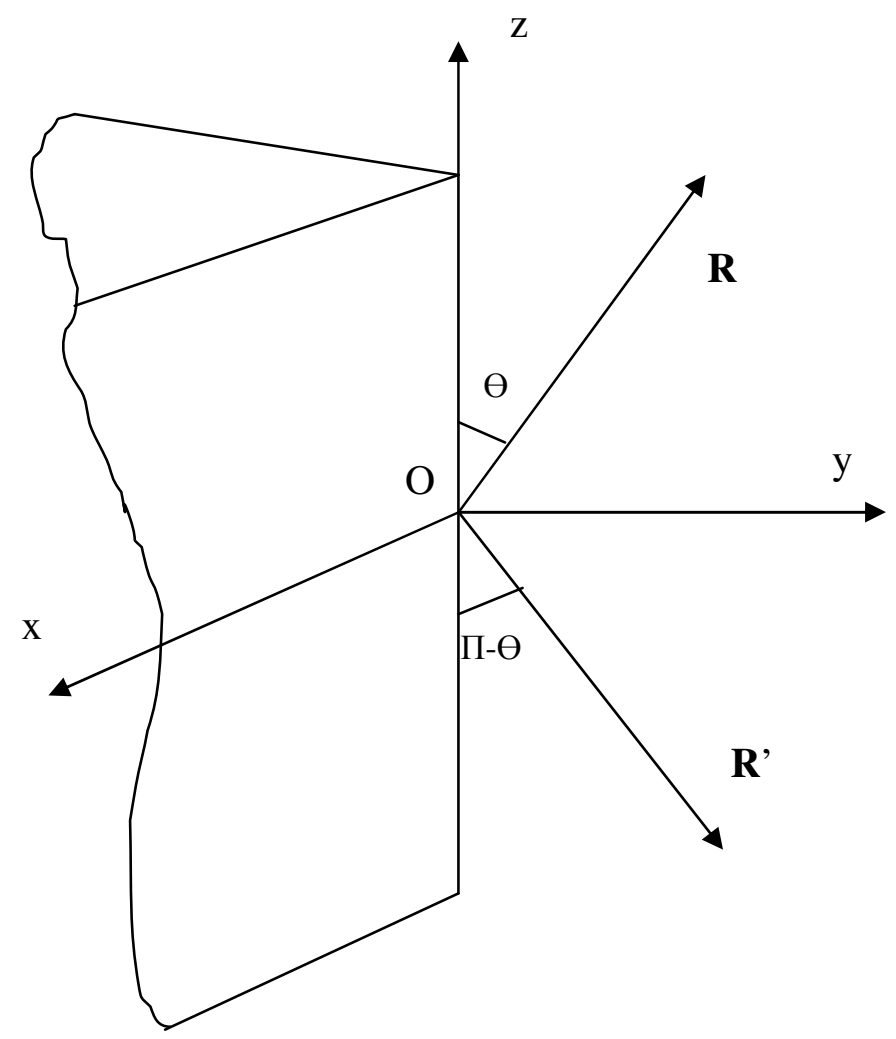

Figure 1. Wedge geometry

\section{Extended UTD}

Let us concentrate on Figure 2. If the electric field of the incident wave is $\bar{E}^{i}=E_{\beta_{o}^{\prime}}^{i} \hat{\beta}_{o}^{\prime}$. Then the $z$-component can be written as:

$$
E_{z}^{i}=-E_{\beta_{o}^{\prime}}^{i} \sin \beta_{o}^{\prime}
$$

By the boundary conditions, the diffracted field should have a z-component given by

$$
E_{z}^{d}=-E_{\beta_{o}}^{i}(0) D_{s} \frac{e^{-j k s}}{s} \sin \beta_{o}
$$

where $\beta_{o}=\beta_{o}^{\prime}$ by generalized Fermat's principle. Since the geometry is infinite in $z$ direction, we can find the other components of the field using Maxwell's equations as follows:

$$
E_{t}^{d}=-j k_{z} \frac{\nabla_{t} E_{z}^{d}}{k^{2}-k_{z}^{2}}+j \omega \mu_{o} \frac{\hat{z} \times \nabla_{t} H_{z}^{d}}{k^{2}-k_{z}^{2}}
$$

Since there is no $z$-component of the $H$ field, only the first term will be used. It is clear from Figure 2 that

One can determine the $\phi$ and $\rho$ components as follows:

$$
k_{z}=-k \cos \beta_{o}
$$

$$
\begin{gathered}
E_{\phi}^{d}=E_{\beta_{o}^{\prime}}(0) \frac{\cot \beta_{o}}{j k \rho} \frac{\partial D_{s}}{\partial \phi} \frac{e^{-j k s}}{s} \\
E_{\rho}^{d}=\frac{\cos \beta_{o}}{j k \sin ^{2} \beta_{o}} \frac{\partial E_{z}^{d}}{\partial \rho}
\end{gathered}
$$




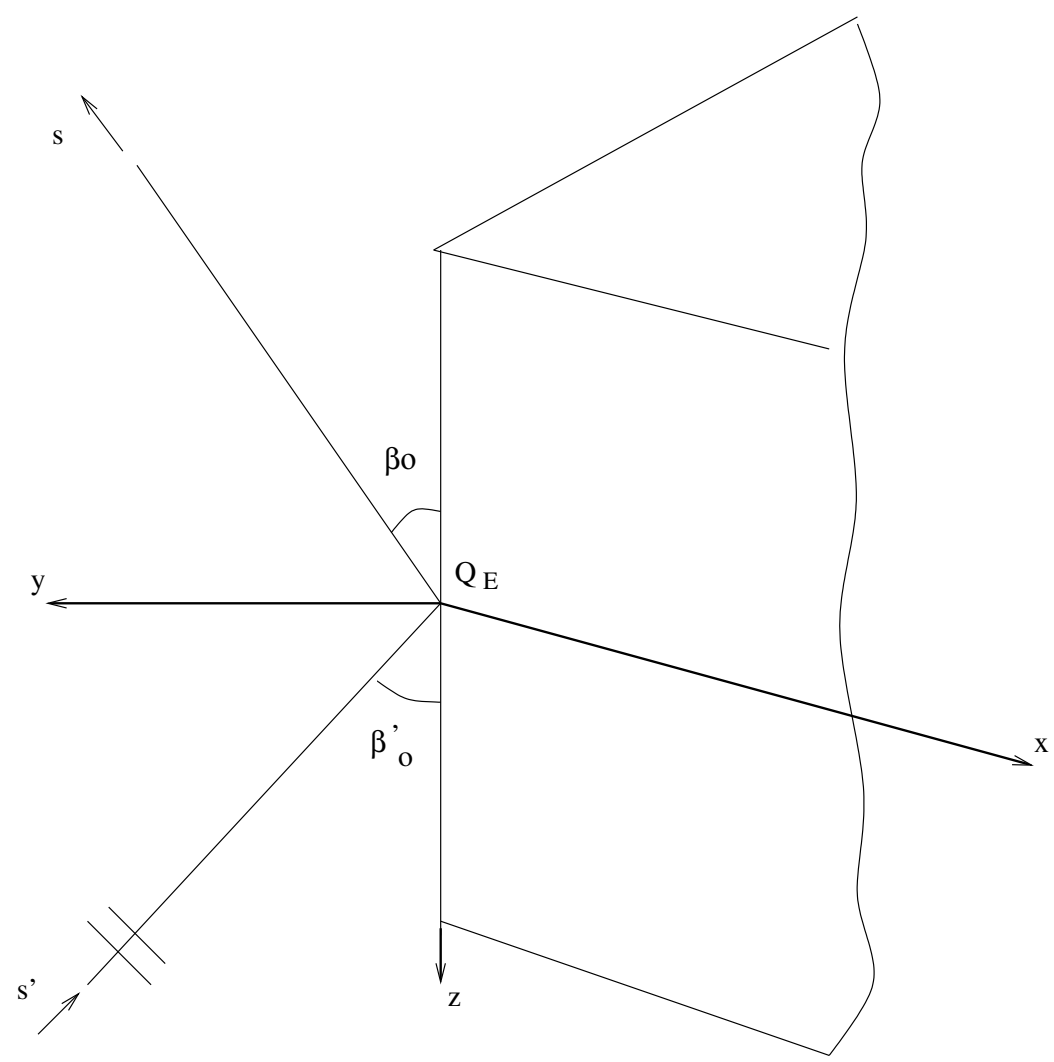

Figure 2. Wedge geometry for the extended UTD

By taking the derivatives carefully, on can write all three components of the diffracted field as follows:

$$
\begin{gathered}
E_{z}^{d}=-E_{\beta_{o}^{\prime}}^{i}(0) D_{s} \frac{e^{-j k s}}{s} \sin \beta_{o} \\
E_{\rho}^{d}=-E_{\beta_{o}}^{i}(0) \frac{e^{-j k s}}{\sqrt{s}} \frac{\cot \beta_{o}}{j k}\left[\frac{1}{\sin \beta_{o}} \frac{\partial D_{s}}{\partial s}-\frac{D_{s}}{2 s \sin \beta_{o}}-j k \sin \beta_{o} D_{s}\right] \\
E_{\beta_{o}}^{d}=E_{\beta_{o}^{\prime}}^{i}(0) \frac{e^{-j k s}}{\sqrt{s}} \frac{\cot \beta_{o}}{j k}\left[\frac{\partial}{\partial s}-\frac{1}{j k}\right] D_{s}
\end{gathered}
$$

A similar approach can made when the electric field of the incident wave is

$$
\begin{gathered}
\bar{E}^{i} \cong E_{\phi^{\prime}}^{i} \hat{\boldsymbol{\phi}}^{\prime} \\
\bar{H}^{i} \cong Y_{o} E_{\phi}^{i} \cdot \hat{\boldsymbol{\beta}}_{o}^{\prime}
\end{gathered}
$$

Combining the results of two polarizations, we can get the following matrix relation: 


$$
\left[\begin{array}{c}
E_{\beta_{o}}^{d} \\
E_{\phi}^{d} \\
E_{s}^{d}
\end{array}\right]=\left[\begin{array}{cc}
-D_{s}+D_{1} & D_{2} \\
D_{3} & -D_{h}+D_{4} \\
D_{5} & D_{6}
\end{array}\right]\left[\begin{array}{c}
E_{\beta_{o}^{\prime}}^{i} \\
E_{\phi^{\prime}}^{i}
\end{array}\right] \frac{e^{-j k s}}{\sqrt{s}}
$$

Here $D_{s}$ and $D_{h}$ are the UTD diffraction coefficients and the other terms are the higher order diffraction coefficients. It is noted that the above result is an exact solution for the diffraction for a half-plane [7].

\section{Conclusion}

Prof. Kouyoumjian is known as the father of UTD. His passing away is a big loss for the electromagnetics community. We are greatly indebted to him for his scientific contributions and gentile personality. We commemorate him on this occasion by giving an example of his latest works in the theory of diffraction. It is also a commemoration for Prof. O. Merih Buyukdura who has passed away in 2007. He was the last PhD student of Prof. Kouyoumjian and had a great potential in electromagnetics as exemplified by his $\mathrm{PhD}$ work.

\section{References}

1. O.M. Buyukdura, "Radiation from Sources and Scatterers near the Edge of a Perfectly Conducting Wedge", PhD dissertation, Ohio State University, Columbus, Ohio, 1984.

2. F. Sitka, "UTD Analysis of Electromagnetic Scattering by Flat Plate Structures", PhD Dissertation, Ohio State University, Columbus, Ohio, 1981.

3. J.B. Keller, “Geometrical Theory of Diffraction”, J. Opt. Soc. Am., vol.52, pp.116-130, 1962.

4. R.G Kouyoumjian, and P.H Pathak., "A Uniform Geometrical Theory of Diffraction for and Edge in a Perfectly Conduction Surface”, Proceedings IEEE, vol.62, pp. 1448-1461, 1974.

5. O.M.Buyukdura, Goad S.D. and R.G. Kouyoumjian, “A Spherical Wave Representation of the Dyadic Green's Function for a Wedge”, IEEE Trans. on Antennas and Propagation, Vol.44, no.1, s.12-22, 1996.

6. J. Van Bladel, "Electromagnetic Fields", McGraw-Hill, New York, 1964.

7. O.M.Buyukdura, "GTD Solution with Higher Order Terms to the Diffraction by an Edge: Towards a Uniform Solution”, IEE Proc.-Microw. Antennas Propagation. vol.143, no.1, s. 43-50, February 1996. 